\title{
Free open access medical education applications: a critical appraisal of techniques for quality assessment and content discovery
}

\author{
Allen Zhi, Abbas Husain \\ Department of Emergency Medicine, Northwell Health Staten Island University Hospital, Staten Island, NY, \\ USA
}

\section{INTRODUCTION}

Free open access medical education (FOAM) applications (apps) enhance traditional reference modalities by exposing users to cutting edge concepts through a dynamic collection of visual and auditory resources which encourage collaboration, information sharing and knowledge translation.

\section{CONTENT DISCOVERY}

FOAM has grown over the past several years from a primarily social media linked means of discussing and disseminating medical education to a multiplatform movement. Among these platforms are medical applications that are accessible on smart phones and tablets, which have become ubiquitous in the healthcare setting. Their use has become increasingly popular, especially amongst young physicians, residents, and medical students.

Similar to other flavors of FOAM, users may initially discover that finding the right apps can be a daunting experience. There are a large number to choose from and at the same time, there is no standard of quality or accuracy to which these apps are held. Quality is based almost entirely on independent user review. A number of these apps are listed at Table 1 with all of them being available on iTunes (https://www.apple.com/itunes) and the android store.

Amongst the established and well-known FOAM websites, few have their own dedicated app such as "WikEM", a Wikipedia-style website for content relevant to emergency medicine. Despite this, most are still accessible through apps via an RSS Feed Reader such as "Read". Though not strictly medical apps, there are a number of these types of apps which function as news aggregators which can sync with FOAM sites and present a wide variety of sources all in one place. These sites, as well as their content creators, can also usually be found on twitter.

Social media style apps specifically for physicians have also cropped up over the past few years. "Figure 1 " is perhaps the most popular with users being able to share pictures and cases with other users, as well as comment and contribute to the discussion. "Daily Rounds" is another app with a cases section where users can comment, as well as containing journal article summaries, clinical guidelines and a wealth of other information. This format of informal discussion
elSSN: 2383-4625

Received: 22 December 2017

Revised: 10 January 2018

Accepted: 17 January 2018

Correspondence to: Abbas Husain Department of Emergency Medicine, Northwell Health Staten Island University Hospital, 475 Seaview Avenue, Staten Island, NY 10305, USA E-mail: abbashu@gmail.com ORCID

https://orcid.org/0000-0001-8750-2473

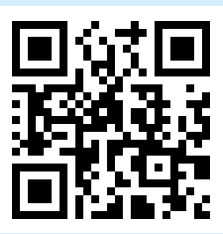

How to cite this article:

Zhi A, Husain A. Free open access medical education applications: a critical appraisal of techniques for quality assessment and content discovery. Clin Exp Emerg Med 2019;6(1):93-95.

This is an Open Access article distributed under the terms of the Creative Commons Attribution Non-Commercial License (http:// creativecommons.org/licenses/by-nc/4.0/). 
Table 1. Select free open access medical education applications and associated homepages

\begin{tabular}{|c|c|c|}
\hline Application & URL & Description \\
\hline Calculate by $0 x M D$ & https://qxmd.com & Clinical calculator and decision support tool \\
\hline Daily Rounds & https://www.dailyrounds.org & $\begin{array}{l}\text { Social network for physicians with clinical references including drug database, case } \\
\text { discussions, calculator and more }\end{array}$ \\
\hline Epocrates & http://www.epocrates.com & Comprehensive drug reference \\
\hline Eye Emergency Manual & $\begin{array}{l}\text { https://www.aci.health.nsw.gov.au/networks/ophthal- } \\
\text { mology/about/eem }\end{array}$ & Clinical reference for emergency ocular complaints \\
\hline Micromedex & http://truvenhealth.com/Products/Micromedex & Comprehensive drug reference \\
\hline One Minute Ultrasound & http://omus.ultrasoundpodcast.com & Quick ultrasound video guides for emergency medicine applications \\
\hline The "Prognosis" App series & http://www.prognosisapp.com & Interactive case scenarios with discussion \\
\hline Resuscitation! & http://www.emgladiators.com/resus & Interactive case scenarios/case simulator with discussion \\
\hline Skyscape & http://www.skyscape.com & Extensive clinical reference including drug database, calculator, news feed and more \\
\hline
\end{tabular}

is akin to formal case reports, but augmented by the ability to discuss the case in real time.

Overall, the most common type of FOAM app is the clinical reference app. Drug references such as "Micromedex" and "Epocrates" are essential tools for any physician. Both feature premium versions with paid content, but their free versions tend to be sufficient the majority of the time. References can range from general resources like "Medscape" to very specific, such as "Eye Emergency Manual," and can be invaluable in the clinical setting. However, a large number of paid clinical reference apps (which have not been listed here) feature more content and more frequent updates than free apps. The best resources for certain content, such as ultrasound, are often still behind a paywall. In spite of this, FOAM in the medical app arena is steadily growing with a constant influx of new apps and even more frequent content updates in existing apps. There is no better time to start exploring.

\section{QUALITY ASSESSMENT}

Only a small number of resources offer standardized assessments of quality of FOAM. ALiEM (Academic Life in Emergency Medicine) attempted to measure quality of FOAM content with the AIR (Approved Instructional Resources) series rubric, ${ }^{1}$ which has been explored in previous articles in this series. ${ }^{2,3}$ Their scoring instrument was used to evaluate blogs and podcasts but in theory can be extended to medical apps and other FOAM content. Recently, a paper from the National University of Singapore ${ }^{4}$ aimed to develop a quality assessment tool for apps that targeted medical-related problems. The first portion heavily skews towards assessing app quality based on pharmacologic applications. However, the second, third, and fourth portions are more generalizable to apps as a whole, evaluating reliability, usability and privacy of apps.

Significant limitations exist in terms of practical applications of these rubrics. First, none of the apps are static. With new evidence and new guidelines being constantly updated in medicine, the developers of these apps have to keep up to date in order to ensure accurate information. In contrast to the traditional textbook, there is no limit to how frequently apps can be and are updated. Second, unlike with podcasts or blogs, a unique challenge of rating these apps is the difference in content and user experience between different platforms. In several instances in the free apps listed above, the same app would have different features or user interfaces on Apple and Android. Lastly, none of these rubrics are readily applicable to the content of social media-style apps. These apps tend to be a forum for discussion. Even if the discussion may be by experts, there is no practical way to assess the quality of information written by authors with a wide range or credentials, or potentially lack thereof.

\section{CONCLUSION}

One of the challenges of working in our increasingly complex healthcare environment is rapidly being able to access accurate and reliable information. With the growing presence of FOAM, especially in the realm of medical apps, information has never before been as readily available at our fingertips. However, with the wide range of content and absence of regulation or standardization, misinformation can just as easily be propagated. In its current state, apps and FOAM at large, although a growing and valuable body of knowledge, should not be used as in a vacuum and is better consumed in conjunction with established resources. 


\section{CONFLICT OF INTEREST}

No potential conflict of interest relevant to this article was reported. No app developers paid me to include their app in this article.

\section{REFERENCES}

1. Chan TM, Grock A, Paddock M, Kulasegaram K, Yarris LM, Lin M. Examining Reliability and Validity of an Online Score (ALiEM
AIR) for rating free open access medical education resources. Ann Emerg Med 2016;68:729-35.

2. Singer AJ, Morley EJ, Mallemat H. What's on tablet PC: an introduction to FOAM at home. Clin Exp Emerg Med 2016;3:181-2.

3. Grock A, Paolo W. Free open access medical education: a critical appraisal of techniques for quality assessment and content discovery. Clin Exp Emerg Med 2016;3:183-5.

4. Loy JS, Ali EE, Yap KY. Quality assessment of medical apps that target medication-related problems. J Manag Care Spec Pharm 2016;22:1124-40. 